\title{
FRACTAL-STRUCTURAL ANALYSIS OF CONVECTION HEAT TRANSFER IN A TURBULENT MEDIUM
}

\author{
Turmukhambetov A.Zh., Aitmanova K.A., Otegenova S.B. \\ al-Farabi Kazakh National University, Almaty,Kazakhstan, Akylbek.turmukhambetov@gmail.com
}

\begin{abstract}
The features of convective heat transfer of bodies in a turbulent environment are considered. The results of experimental research by one of the authors are discussed. Experimental data show that the heat transfer of a spherical body is affected by natural convection, the thermo-physical properties of the medium, the tightness of the flow, the turbulent flow regime, etc. Due to these factors, the formula for calculating convective heat transfer, which includes many experimental constants, becomes cumbersome and inconvenient for practical application. The paper presents the results of applying fractal-structural analysis methods to describe experimental data on convective heat exchange of badly streamlined (cylinder and sphere) bodies in a channel. Quantitative relations are obtained that link the intensity of turbulent heat transfer with the criteria for the degree of self-organization.
\end{abstract}

Keywords: turbulence, heat transfer, fractal, multifractal, vortex structure, entropy, flow crampedness.

\section{Introduction}

Analysis of numerous turbulence models shows their limitations and lack of accuracy. In many cases, to reach agreement with the experiment, the method of fitting using multiple empirical coefficients is used, or various empirical functions are introduced that are not physically justified. This approach may be useful for describing a specific process, but it is not sufficient for developing more or less General methods for solving turbulence problems. There is an opinion about the need for a deeper study of the physics of turbulent exchange to create an adequate theory, which puts turbulence in the category of General physical problems, and the results obtained in this direction are of General scientific significance. To understand the physics of hydrodynamic turbulence, it is important and relevant to conduct a theoretical study based on simple and visual models (for example, fractal and multifractal) of the dynamics of interaction of structural elements vortices and vortex clusters in gradient flows, paying special attention to convective heat transfer in homogeneous and heterogeneous media, as a possibility of tracking the evolution of turbulent motion. At the same time, it is obvious that the value of any theoretical model depends on the degree of coincidence with the results of well-known or specially designed experiments.

\section{Experimental results}

Figures 1-3 show data on the average convective heat transfer of a sphere in a constrained flow, which shows a complex dependence of the heat transfer intensity on natural convection, the thermo-physical properties of the medium, and the degree of flow congestion. More than 1000 experiments were carried out to measure the convective heat exchange of a sphere flowing around a viscous liquid flow in a channel with a wide range of changes in the experimental conditions: the degree of flow constraint, the magnitude and direction of natural convection, the directions of heat flow, and the thermo-physical properties of the medium. A description of the experiment and part of the results are presented in [1,2]. As you know, to date, we have observed the desire of the authors of such studies to present the results of heat exchange in the form of formulas:

$$
N u=a R e^{b}+c R e^{d},
$$

where $a, b, c, d$ are empirical constants that take into account the above and other similar conditions of experience.

In any case, the dependence of heat transfer on the values of the Reynolds number is clearly shown, which can be represented as:

$N u=C R e^{n}$.

Obviously, the explicit form of formula (1) depends on the flow mode: laminar or turbulent. In this regard, it should be noted that while some of the experimental results we have studied are known to relate to 
the laminar regime, most of them relate to the transient and turbulent flows. Although it is generally assumed that turbulence occurs when the sphere flows around $\mathrm{Re} \geq 40$, it seems that anisotropic turbulence is observed much earlier. It should be noted that in the case of heat exchange, non-isothermicity is an additional factor that introduces a disturbance in the flow.

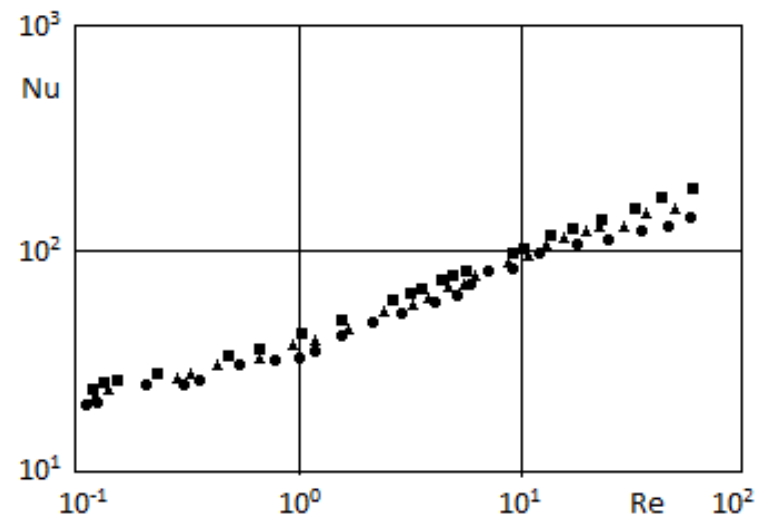

Fig.1. Influence of natural convection on the average heat transfer of the sphere, $\operatorname{Pr}_{\mathrm{f}}=6120$. Designations: • $-\mathrm{Gr}=13.0 ; \boldsymbol{\Lambda}-\mathrm{Gr}=20.0 ; \boldsymbol{-}-\mathrm{Gr}=38.0$.

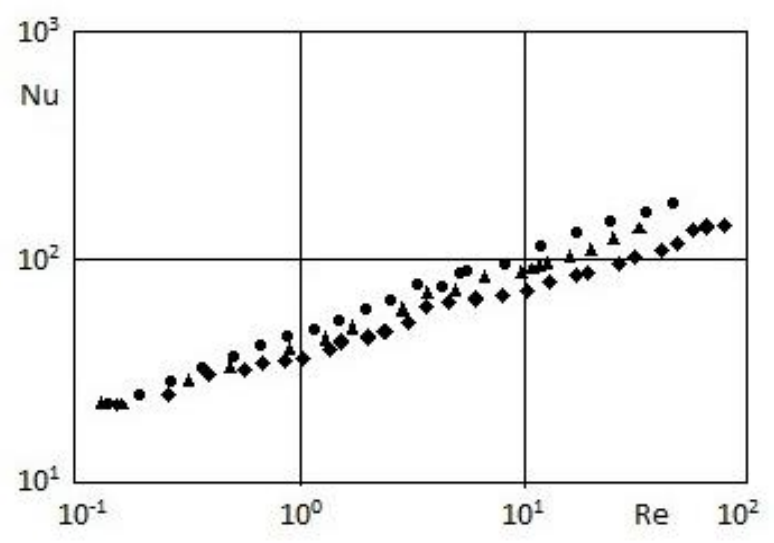

Fig.2. Influence of the thermo-physical properties of the medium on the heat transfer of the sphere. $\operatorname{Pr}_{\mathrm{w}} / \mathrm{Pr}_{\mathrm{f}}=0.20$. Designations: $\bullet-\operatorname{Pr}_{\mathrm{f}}=8680$; $\operatorname{Pr}_{\mathrm{f}}=6120 ;-\operatorname{Pr}_{\mathrm{f}}=3080$



Fig.3. Influence of restricted flow on convective heat transfer of the sphere. Designations: $\mathbf{\Delta}-\chi=0.235 ; \bullet-0.148$

Thus, as a result of the experiments, systematic data on the hydrodynamic resistance and heat exchange of a sphere in the region of relatively small Reynolds numbers were obtained for the first time. Under the accepted experimental conditions, it was possible to ignore the influence of natural convection already at $\mathrm{Re}$ $\geq 10$, paying attention to the flow constraint as the main factor leading to inhomogeneity of the turbulent flow. In article, the degree of flow constraint, which determines the spatial scales of complex turbulent eddy formations both on the surface of the body and a track of the body, is calculated as the change in the area of the gap between the surfaces of the streamlined body and the limiting walls of the channel. The geometrical parameters of the problem of fluid flow around a cylinder and a sphere in a channel are shown in Fig. 4.

The degree of crampedness of the flow is expressed by the number $\chi=h / H$, where $h$ and $H$, respectively, are the characteristic dimensions of the body and channel (in our case, the diameters). Therefore, $0 \leq \chi<1$. Limiting case $\chi=0$ corresponds to the flow of an unlimited fluid flow around a body. The relative values of the gap area in the midsection sections are defined as

$$
\frac{F_{01}-F_{1}}{F_{01}}=1-\chi, \quad \frac{F_{02}-F_{2}}{F_{02}}=1-\chi^{2},
$$

where $F_{01}, F_{02}$ - the cross-sectional area of the channel and pipe, $F_{1}, F_{2}$ - the median section area of the respectively cylinder and sphere. 
The movement in the gap between the body surface and the channel wall is inhomogeneously turbulent. Therefore, strictly speaking, the structural elements of turbulence are self-affine (not self-similar) multifractals that have different similarity coefficients in the corresponding spatial directions. One of the consequences of this complication of fluid movement in a limited space is that now the effective gap area-the characteristic size of the fluid flow is smaller than both in laminar motion and incomparison with the case of free turbulence. This will lead to an additional increase in the coefficient of hydrodynamic resistance of the body and the intensification of the heat exchange process.

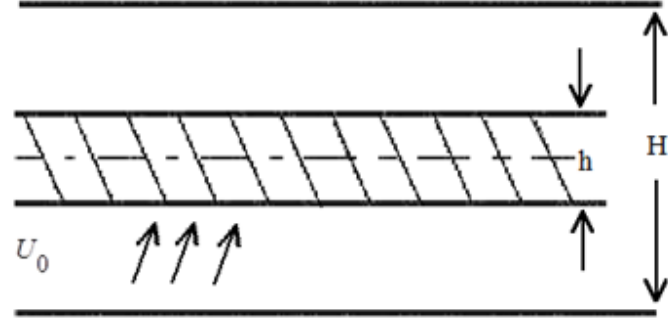

$a$

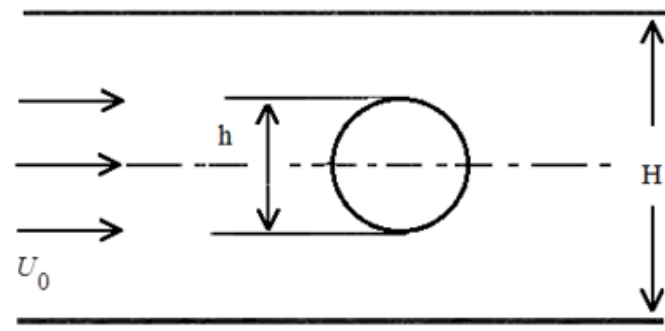

$b$

Fig.4. Flows diagram of the cylinder in the channel (a), and the sphere in the pipe (b)

Obtained experimental data on the heat exchange of the sphere in the channel confirm the structurality of the flow even at relatively small Reynolds numbers. For the first time, the natural extension of the known regularities to the region of small Re numbers (up to the obviously laminar regime) allows us to consider our experimental data as test data confirming the provisions of the proposed structural approach to the study of convective heat transfer in a turbulent environment.

\section{Theoretical part}

Development by Mandelbrot [3] the mathematical concept of a fractal and its applications to the description of the shapes of various objects make it possible to construct models of non-trivial random scaleinvariant structures $[4,5]$. The application of these models is a new approach to the description of disordered structures in physics. Success in applying fractal models in physics is primarily due to the fact that fractal forms are inherent in a huge number of processes and structures. Especially successful the possibilities of theory fractal are used especially successfully in studies of hydrodynamic turbulence and heat transfer [6-9].

The fractal character of hydrodynamic turbulence follows from the scale-invariant property of turbulence dynamics and from the structure of a strange attractor corresponding to the occurrence of hydrodynamic chaos. The structural and scaling characteristic of the evolution of turbulent vortices allows us to consider them as a fractal object. Moreover, numerous experimental studies show that turbulence, as a combination and result of the interaction of different-scale vortices, is a multifractal characterized by a set of different fractal dimensions. The dimension of the multifractals itself or the generalized dimension $D_{q}$ is determined by the Renyi formula [4]:

$$
D_{q}=\frac{1}{q-1} \lim _{\delta \rightarrow 0} \frac{\ln N \delta, q}{\ln \delta},
$$

where $-\infty \leq q \leq+\infty \quad$ - is order of the multifractal moment, $N \delta, q \quad$ - is number of vortices.

It is well-known, the multifractality of turbulence is directly related to its intermittency. Representing the characteristic of the dependence of the number of vortices on their size in the form:

$$
N q, \delta \sim \delta^{-\tau q} ; \quad \tau q=-\lim _{\delta \rightarrow 0} \frac{\ln N \delta, q}{\ln \delta}
$$

can be received:

$$
D_{q}=\frac{1}{1-q} \tau q
$$

where $\tau q$-is the intermittency function, which can be determined from the experiment by processing the time series. 


\subsection{Fractal nature of convective heat transfer}

Indicators of power dependencies between dimensionless complexes of basic parameters (similarity criteria) can be expressed in terms of fractal characteristics determined by theoretical models of structures (vortices, clusters of particles) of the phenomenon. We present the Nusselt criterion for turbulent heat transfer as:

$$
N u=\frac{\alpha l_{0}}{\lambda}=\frac{q}{q_{0}} ; \quad \alpha=\frac{q}{\Delta T} ; \quad q_{0}=\lambda \frac{\Delta T}{l_{0}},
$$

where $\alpha-$ is the heat transfer coefficient; $l_{0}$ - is the characteristic spatial scale; $\lambda-$ is the thermal conductivity; $q, q_{0}$ are the heat flows through the unit surface at the temperature difference $\Delta T$ in turbulent and laminar modes.

The $q / q_{0}$ ratio is equivalent to the ratio of the effective surface area of turbulent mixing $F$ to its value $F_{0}$ in laminar mode. The law of increasing $F$, which corresponds to the intensification of heat exchange during flow turbulence, according to $[10,11]$ can be represented in the most general form as power dependence:

$$
F=F_{0}\left(\frac{\ell_{0}}{\ell_{v}}\right)^{\gamma},
$$

and equation (3) goes to the form:

$$
N u=\frac{q}{q_{0}}=\frac{F}{F_{0}}=\left(\frac{l_{0}}{\delta_{v}}\right)^{\gamma},
$$

where $l_{0}-$ the minimum dissipative scale is replaced by $\delta_{v}$ - the smallest size of self-similar structural elements of turbulence, depending on the kinematic viscosity $v ; \gamma-$ an indicator determined by a specific scaling model - scale-invariant deformation of the surface of the medium structures.

Considering the well-known estimate $\delta_{v} \sim v / U_{0}$ from formula (4), we obtain

$$
N u=C R e^{\gamma},
$$

where $R e=l_{0} U_{0} / v$ - the Reynolds number make up the characteristic velocity $U_{0}, C$ is a constant.

Some fixed values of $\gamma$ can be determined from simple models of scale-invariant deformation of surfaces that restrict liquid volumes. It should be expected that isotropic turbulence actually corresponds to symmetric deformation, and anisotropic turbulence, which is more typical for shifted flows, corresponds to one - sided asymmetric deformation. It is taken into account that model deformations have the properties of turbulent mixing: maximum surface stretching with the smallest number of links, self - similarity of the cascade deformation process-the next deformable surface (generator) must contain parallel elements to the original surface. The value of $\gamma$ can be found by calculating the fractal (Hausdorff) dimension $D$ of the object obtained after the $\mathrm{n}$-th deformation. By definition

$$
D=\lim _{l_{n} \rightarrow 0} \frac{\ln N\left(l_{n}\right)}{\ln 1 / l_{n}}, \quad N\left(l_{n}\right)=F_{n} / l_{n}^{d-1},
$$

where $N\left(l_{n}\right)$ - is the number of elements required to cover the surface $F_{n} ; d$ - is the topological dimension of the space in which the object with the deformable surface is insirted.

As a result of simple calculations, we find a formula that relates the scaling exponent $\gamma$ to the fractal dimension $D$ :

$$
\gamma=D-d+1 \text {. }
$$

Substituting the values $D$ in (6) and taking into account that for isotropic and anisotropic deformations $d$ is equal to 3 and 2, respectively, we calculate the founded values: $\gamma: \gamma_{0}=0.7925$ and $\gamma_{\perp}=0.4650$. Finding from geometrical consideration index of $\gamma$ process scaling should probably be considered as their limit values describing the universal properties of turbulent transport. In particular, $\gamma_{0}$ corresponds to the region of large Reynolds numbers, when almost homogeneous, isotropic (Kolmogorov) turbulence is observed, $\gamma_{\perp}$ can be used both for large Reynolds numbers, when the anisotropy is caused by local (boundary) conditions (the presence of walls, the second phase, heat transfer, etc.), and for relatively small Re numbers, i.e. in the processes of occurrence and evolution of turbulence. The calculated values of $\gamma_{\mathrm{o}}, \gamma_{\perp}$ are close to the well- 
known experimental results on heat exchange for developed, isotropic turbulence $(\mathrm{n} \sim 0.8)$ and for quasiregular, large-scale turbulence $(\mathrm{n} \sim 0.5)$.



Fig.5. Average cylinder heat transfer. The results are taken from [1]. 1 - dat of J. Ulzamer; 2 - A.A. Zukauskas; 3 B.Kh. McAdams; 4 - M.A. Mikheev and I.M. Mikheeva; 5 - G. Hilpert

Figure 5 shows a comparison of numerous results of various authors presented in [1] in the form of empirical dependencies with the formula (5) for two limit values $\gamma$. As can be seen from the graph, the theoretical dependence (5), covering the lower and upper regions of the values of the number Re, correctly reflects the laws of heat exchange of the cylinder under different flow modes. Therefore, instead of an empirical dependence (1) with an undefined degree $n$, we propose the formula (5), where the exponent $\gamma$ is associated with the fractal dimension $D$. The indexes $\gamma$ of the dependences degree of turbulent heat transfer can be determined not only by geometric, but also by physical - multi-fractal models connected with information-entropy concepts.

\subsection{Multifractal (physical) model of turbulent heat transfer}

The movement of turbulent vortices of different scales, their interaction is observed as random alternations of different-scale dynamic characteristics. Therefore, it is necessary to take into account the probabilistic, intermittent nature of turbulent heat transfer. Objects in which the distribution of a measure is described by probabilistic moments of different order (the degree of inter leavability) and the corresponding spectrum of fractal dimensions are called multifractals. In [4], the following is one of the definitions of the multifractal probability measure $M$ :

$$
M \sim \delta^{-\alpha} N(q, \delta) ; N(q, \delta)=\sum_{i} P_{i}^{q} \sim \delta^{q D_{q}} \times \delta^{-D_{q}}=\delta^{D_{q}(q-l)}=\delta^{-\tau(q)},
$$

where $\delta$ - size of the measure split cell, $\alpha$ - fractal dimension of the cell, $N(q, \delta)$ - number of cells containing the measure, $P_{i}$ - probability of realization cell number $i, q$ - the order of the multifractal moment, which takes any real value, $\tau(q)=(1-q) D_{q}$-interleaving function, $D_{q}$ - a generalized (multi-fractal) dimension defined by the Renyi formula, which differs from (2) in that instead of $N(q, \delta)$ - the number of vortices, we consider $P_{i}$ - the probability of their realization:

$$
D_{q}=\frac{1}{q-1} \lim _{\delta \rightarrow 0} \frac{\ln \sum_{i} P_{i}^{q}}{\ln \delta} .
$$

$N(q, \delta)$ it can also be expressed via local characteristics

$$
N(q, \delta)=\sum_{i} P_{i}^{q} \sim \delta^{q \times \alpha} \times \delta^{-f(\alpha)}=\delta^{q \alpha-f(\alpha)},
$$

where $f(\alpha)$ is multifractal spectral function.

From (7) and (9) we obtain an unambiguous cyclic relation between the functions $\tau(q)$ and $f(\alpha)$ via Legendre transformations: 


$$
f(\alpha(q))=q \times \alpha(q)+\tau(q)
$$

where $\alpha(q)=-\frac{d \tau(q)}{d q}, \quad q=\frac{d f(\alpha)}{d \alpha}$.

In the case of $q=1$, there is no interleaving (by definition $\tau(1)=0$ ) and the chaos structures can be selfsimilar. If we reveal the uncertainty in (8) that occurs when, $q \rightarrow 1$ by decomposing the numerator with a small argument $q$ - 1 , we have

$$
D_{l}=\lim _{\delta \rightarrow 0} \frac{\sum_{i} P_{i}(\delta) \ln P_{i}(\delta)}{\ln \delta}=-\lim _{\delta \rightarrow 0} \frac{S(\delta)}{\ln \delta}=S ; \quad S(\delta)=-\sum_{i} P_{i} \ln P_{i},
$$

where $S(\delta)$ - entropy of splitting a measure into cells, $S$ - is the entropy of a self-similar set that does not depend on splitting.

From formulas (10), (11) follows

$$
f\left(\alpha_{1}\right)=\alpha_{1}=D_{1}=S, \quad \alpha_{1} \equiv \alpha(q=1) .
$$

In short, the entropy of a self-similar set is equal to the value of the multifractal spectral function at its fixed point. Information entropy, defined by the second expression (11), according to the generally accepted terminology [12] is the average value of synergetic information $I_{i}$, acquired at the construction (destruction) of the structure with probability $P_{i}$

$$
I_{i}=-\ln P_{i} ; \quad \sum P_{i}=1 \text {. }
$$

We will represent the probability of realization of structures $P$ as a function of a continuous variableinformation $I[13]$ :

$$
P(I)=e^{-I}, \quad \int_{0}^{\infty} P(I) d I=1 .
$$

The probability $P(I)$ is expressed through the probability density distribution function $f(I)$

$$
P(I)=e^{-I}=\int_{I}^{\infty} f(I) d I,
$$

where the limits of integration correspond to areas of definition $P(I) \geq 0$.

Relation (12) is satisfied by the function

$$
f(I)=P(I)=e^{-I}, \quad \int_{0}^{\infty} f(I) d I=1 .
$$

The probability function of the realization of information $P(I)$ coincides with the probability density function $f(I)$. Precisely information is an integral characteristic of a self-similar system inherent in any of its hierarchical levels: a part contains complete information about the whole. Taking into account (13), information of entropy of self-similar systems we write in the form:

$$
S(I)=-\int_{I}^{\infty} f(I) \times I d I=(I+1) e^{-I} .
$$

Further, according to [14], any continuous function $g(x)$, describing self-similar properties of dynamic chaos at its fixed point satisfies the functional equation:

$$
g(x)=\alpha g(g(x / \alpha))
$$

where $\alpha-$ is a scaling multiplier. Therefore, as criteria for self-similarity, we take the values of the characteristic functions of probability $P(I)$ and information entropy $S(I)$ at their fixed points:

$$
\begin{aligned}
& P\left(I_{1}\right)=e^{-I_{I}}=I_{1}, \quad I_{1}=0.567, \\
& S\left(I_{2}\right)=\left(I_{2}+1\right) e^{-I_{2}}=I_{2}, \quad I_{2}=0.806 .
\end{aligned}
$$

Taking into the account the taken results from the formula (5) for the intensity of heat transfer in the developed turbulent regime $(\tau(q=1)=0)$ we write in the following way: 


$$
N u=C R e^{I_{2}},
$$

where $C$ - is a new constant.

Similarly, for anisotropic turbulence

$$
N u=C R e^{I_{I}}
$$

The numbers $I_{1}, I_{2}$ are extended analogues of the Fibonacci number, which is used in many areas of modern science, and correspond to two extreme cases of describing the conduct of a complex system: informational and entropy. Between statistics and dynamic conditions realizes a informational state (the beginning of structuring and stochastization), described by a number $I_{I}=0.567$. The most complex statistical state with internal order is characterized by a number $I_{2}=0.806$ - the value of entropy, normalized to unit. The numbers $I_{l}, I_{2}$ they can be considered as the minimum values of the fractal dimensions of the set of values of information generated by the system at qualitatively different levels of its evolution. In a broad sense, numbers $I_{l}, I_{2}$ are universal quantitative criteria for qualitative changes in the evolution of open systems of any nature.

\section{Discussion}

\subsection{Influence of flow tightness on average heat exchange.}

From the analysis of the data shown in figures 1-3, follows that the main factor affecting the heat exchange of the body in the channel is the tightness of the flow. Obviously, the mechanism of influence of the constraint factor also works in the case of flow around bodies without heat exchange. The study of fractal properties of a constrained turbulent flow provides the following correction for changes in the hydrodynamic resistance of poorly streamlined bodies [15]:

$$
R e_{\chi}=\frac{R e_{m}}{\left(1-\chi^{j}\right)^{D_{i} / 2}},
$$

where the index « $\chi$ » - means that Re is calculated adjusted for tightness; $R e_{m}$ - the Reynolds number calculated from the maximum speed; $j=1$ corresponds to a cylinder, $j=2$ - a sphere; $D_{i}$ is calculated using the formula (6), where $\gamma_{1}=\gamma_{\perp}=0.4650, \gamma_{2}=\gamma_{0}=0.7925$.

Therefore, taking into account (18), formulas (16) and (17) can be given the form:

$$
N u=C\left[\frac{R e_{m}}{\left(1-\chi^{j}\right)^{D_{i} / 2}}\right]^{I_{i}} \text {. }
$$

Figure 6 shows the results of processing experimental data on the heat exchange of the sphere (the experimental conditions and initial data are shown in figure 3). The coincidence of the values of $\mathrm{Nu}$ calculated by formula (19), where for a specific case it is accepted: $j=2 ; D_{l}=2.465 ; I_{i}=I_{1}=0.567$, confirms the fruitfulness of applying the fractal theory to the study of heat transfer (hence, hydrodynamics) of a body flowing around a constrained flow of a viscous liquid.

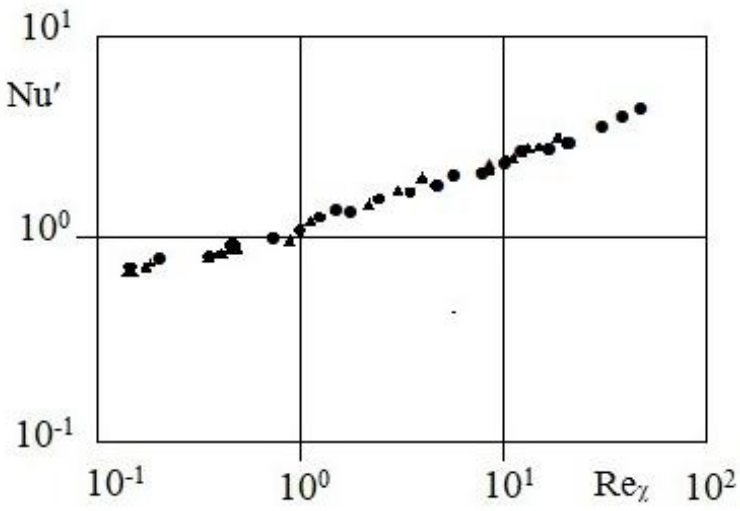

Fig.6. Taking into account the effect of flow constraint on convective heat exchange of the sphere. The designations and conditions of the experiment correspond to fig. 3

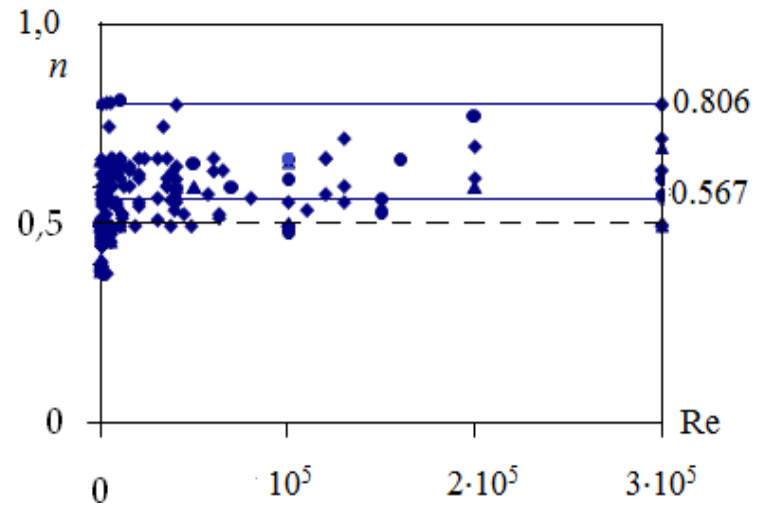

Fig.7. Average heat transfer of bodies in a viscous fluid flow. Designations: - experimental data of various authors for the cylinder [14], $\bullet-$ for the sphere [1]. 


\subsection{Average heat exchange of bodies in a turbulent flow.}

The obtained theoretical results are compared with the known experimental data on convective heat exchange of bodies (figure 7). The results of more than 100 publications are analyzed, the main part of which is given in [16]. Experiments cover a wide range of factors (various liquids and gases, sizes and shapes of bodies, the range of changes in the number of Re, research methods, etc.) that affect the course of the heat exchange process and the accuracy of the study itself. At first glance, there is actually a significant spread of the indicator $n$ both in terms of the Reynolds number and for the same value. But this scatter is apparent: if we do not take into account the results for the smallest Reynolds numbers, when the values of $n \leq 0.5$ are selected based on the theoretical solution for laminar flow, the remaining data are located in the interval $I_{1} \leq n \leq I_{2}$.

\section{Conclusions.}

The given results of an experimental research of the average heat transfer of a sphere in a constrained turbulent flow. Here proposed simplified calculation formulas for heat exchange of bodies in a channel with a smaller number of empirical constants. On the basis of results measurements and theoretical analysis is confirmed the fractal nature of convective heat transfer. Presented a fractal description of the influence of the constraint factor on the convective heat exchange of the body. Set up information-entropy criteria for the degree of self-organization of the heat exchange process. Comparison of the numerical results obtained on the basis of the proposed fractal models with the known experimental data shows their satisfactory agreement. The results discussed complement the known information about heat exchange in a turbulent environment, and can be directly used in technological processes related to flow and heat exchange, in order to control and manage them.

\section{REFERENCES}

1 Isataev S.I., Akylbaev Zh.S., Turmukhambetov A.Zh. Aerohydrodynamics and heat exchange of curved bodies. Almaty, Gylym, 1996, 437 p. [in Russian]

2 Turmukhambetov A.Zh. Heat Transfer of a sphere in a constrained flow of a viscous liquid. IFJ. 2001, Vol. 74, No.3, pp. 161 - 163. [in Russian]

3 Mandelbrot B.B. Fractals: form, chance and dimension. San Francisco, 1977, 347 p.

4 Feder E. Fractals. Moscow, Mir, 1991, 254 p.

5 Mandelbrot B. Fractal geometry of nature. Moscow, 2002, 656 p.

6 Kolesnichenko A.V., Marov M. Ya. Turbulence and self-organization. Problems of modeling space and natural environments. Moscow, 2009, 632 p. [in Russian]

7 Kovalnogov V.N., Khakhalev Yu.A. Numerical investigation of effected turbulent flow on the base of pressure fluctuations fractal dimension analysis. Vector of science of Tolyatti state University. 2014, No. 3 (29), pp. 62 - 66. [in Russian]

8 Suprun T.T. Heat transfer in the presence of transition induced by wakes of hesitating cylinder. Eurasian Physical Technical Journal, 2016 Vol. 13, No. 2(26), pp. 92 - 97.

9 Demenok S.L. Heat transfer and hydraulic resistance in pipes and channels. SPB, N-PromByuro, 2012,285 p. [in Russian]

10 Stern V.. Elementary structural model of turbulent mixing. In: Collection of Structural turbulence. Novosibirsk. 1982, 166 p. [in Russian]

11 Zhanabaev Z.Zh. Fractal model of turbulence in a jet. Izvestia of SB as USSR. Series of technical Sciences. 1988, Vol. 4, No. 15, pp. 57 - 60. [in Russian]

12 Haken G. Information and self-organization. Moscow, Mir, 1991, 240 p.

13 Zhanabaev Z.Zh. Information properties of self-organizing systems. Reports of the national Academy of Sciences of the Republic of Kazakhstan, 1996, No. 5, pp. $14-19$.

14 Shuster G. Deterministic chaos. Moscow, Mir, 1988. - 240 p.

15 Turmukhambetov A.Zh. Fractal properties of the constrained turbulent flow. Bulletin of the NAS RK. Almaty, 1999, No. 5, pp. 77 - 83. [in Russian]

16 Morgan V.T. The overal convective Heat Transfer from smooth circular cylinders. Advances in Heat Transfer. 1975, Vol. 11, pp. $199-264$. 